\title{
The clinical effect of plum blossom needle acupuncture with qi-invigorating superficies-consolidating therapy on seborrheic alopecia
}

\author{
Qiuyi $\mathrm{Li}^{1}$, Yubin Xie ${ }^{1}$, Xushan Zha ${ }^{2}$ \\ ${ }^{1}$ First Clinical Medical College of Guangzhou University of Chinese Medicine, Guangzhou 510405, China; ${ }^{2}$ Department of Dermatology, The First \\ Affiliated Hospital of Guangzhou University of Chinese Medicine, Guangzhou 510405, China \\ Contributions: (I) Conception and design: Q Li; (II) Administrative support: X Zha; (III) Provision of study materials or patients: X Zha; (IV) \\ Collection and assembly of data: Q Li, Y Xie; (V) Data analysis and interpretation: Q Li, Y Xie; (VI) Manuscript writing: All authors; (VII) Final \\ approval of manuscript: All authors. \\ Correspondence to: Xushan Zha. Department of Dermatology, the First Affiliated Hospital of Guangzhou University of Traditional Chinese Medicine, \\ Guangzhou 510405, China. Email: lmmengmeng@126.com.
}

Background: Seborrheic alopecia (SA) is a common dermatological disease with a long disease course, and treatment for this disease usually exhibits slow effects. Currently, Western medicine treatments have shown some effects; however, they also have certain limitations. In recent years, Chinese medicine has made breakthroughs in treating SA. The efficacy of plum blossom needle acupuncture with qi-invigorating superficies-consolidating therapy for SA was observed, and its clinical effects were investigated in this study.

Methods: A total of 87 patients with SA treated at the First Affiliated Hospital of Guangzhou University of Chinese Medicine from September 2018 to September 2019 were enrolled as the research subjects. They were divided into a Western medicine group and a Chinese medicine group by the random number table method. The 43 patients in the Western medicine group were treated with conventional Western medicine, and the 44 patients in the Chinese medicine group were treated with a comprehensive traditional Chinese medicine regimen of plum blossom needling with qi-invigorating superficies-consolidating therapy. The treatment effects, changes in estradiol (E2) and testosterone (T) levels, and scores for various body signs (hair growth and hair loss) of the two groups before and after treatment were compared.

Results: The difference in total effective rate between the Chinese medicine group and the Western medicine group (95.45\% vs. $81.40 \%)$ was statistically significant $(\mathrm{P}<0.05)$. After treatment, the $\mathrm{T}$ levels of both groups were lower than before treatment, and the E2 levels of both groups were higher than before treatment; the difference between the two groups was statistically significant $(\mathrm{P}<0.05)$. After treatment, the hair growth and hair loss scores of the two groups were lower than those before treatment, and those of the Chinese medicine group were lower than those of the Western medicine group; the differences were statistically significant $(\mathrm{P}<0.05)$.

Conclusions: Plum blossom needle acupuncture combined with qi-invigorating superficies-consolidating therapy is significantly effective for treating patients with SA. It can effectively adjust the patient's hormone levels, improve hair loss, and promote hair growth. It has the advantages of simplicity, tolerability, and low cost. It cures the cause of the disease and is worth promoting.

Keywords: Plum blossom needle; qi-invigorating superficies-consolidating therapy; seborrheic alopecia (SA); efficacy

Submitted Mar 10, 2020. Accepted for publication Apr 27, 2020.

doi: 10.21037/apm-20-909

View this article at: http://dx.doi.org/10.21037/apm-20-909 


\section{Introduction}

Seborrheic alopecia (SA), also known as androgen-induced baldness and premature baldness, is a type of hair loss caused by excessive seborrhea (1). Patients with this disease have notable scalp seborrhea and significant itching, often accompanied by excessive dandruff, greasy hair and other symptoms. The disease is more common in men than in women (2). In the early stage, hair loss often starts from both sides of the forehead and gradually extends to the top of the head, which seriously affects the patients' appearance and aggravates their psychological pain. The disease is a worldwide problem (3). Western medicine treatments have shown some effects; however, they will produce certain side effects. For example, finasteride may reduce sexual function, and minoxidil may cause allergies and hirsutism. It falls within the severe range of "insect bitten alopecia androgenetic alopecia" in traditional Chinese medicine, and it is believed that this disease is significantly related to the viscera, qi and blood. Plum blossom needle acupuncture is an important treatment method in traditional Chinese medicine that has the advantages of low cost, high tolerance, convenience, and minimal adverse reactions. The qi mentioned by Chinese medicine has the effect of promoting and solidifying. The method of qi-invigorating superficies-consolidating is to stimulate the scalp function by replenishing the qi of the spleen and kidney, so as to solidify the hair and prevent detachment. To investigate the clinical effects of plum blossom needle acupuncture with qi-invigorating superficiesconsolidating therapy in the treatment of SA, 87 patients with SA who were treated at our hospital from September 2018 to September 2019 were enrolled as research subjects. The detailed report is as follows. We present the following article in accordance with the STRICTA-CONSORT Extension for Acupuncture Checklist (available at http:// dx.doi.org/10.21037/apm-20-909).

\section{Methods}

\section{General information}

A total of 87 patients with SA treated at our hospital from September 2018 to September 2019 were enrolled as research subjects and were divided into a western medicine group and a Chinese medicine group by the random number table method. It is to use SAS software to make a random table, and then assign a random number according to the order of visits. There were 43 patients in the Western medicine group and 44 patients in the Chinese medicine group. In the Western medicine group, there were 31 males and 12 females aged 23 to 58 years (average age $33.53 \pm 5.32$ years) whose disease courses ranged from 3 to 35 months (average disease course $15.68 \pm 3.25$ months). In the Chinese medicine group, there were 32 males and 12 females aged 24 to 57 years (average age $33.62 \pm 5.28$ years) whose disease courses ranged from 3 to 36 months (average disease course $15.59 \pm 3.16$ months). The general characteristics of the two groups were not significantly different $(P>0.05)$; thus, the two groups were comparable.

\section{Inclusion and exclusion criteria}

\section{Inclusion criteria}

Patients met the standards described in "Dermatology of Integrated Traditional Chinese and Western Medicine" by Zhili Zhang (4); the disease had not been treated with other medication in the past two months, and the patients had no allergies to the drugs involved in this study. The patients volunteered to participate in this study, and the study was approved by our ethics committee.

\section{Exclusion criteria}

Patients with a history of allergy to the drugs used in this study were excluded, as were those with combined secretory alopecia, drug-induced hair loss, or congenital alopecia areata; those with combined bacterial infections of the scalp or tinea capitis; those with previous hair transplant surgery; and those with hematopoietic, kidney, liver, heart, or brain diseases (5).

\section{Study methods}

The 43 patients in the Western medicine group were given a conventional Western treatment of oral cystine tablets (approval number: Sinopharm standard H32025797; production unit: Yangzhou Aidi Pharmaceutical Co., Ltd.) $50 \mathrm{mg} 3$ times/day and vitamin B6 tablets (approval number: Sinopharm standard H44024264; production unit: Guangzhou Baiyunshan Guanghua Pharmaceutical Co., Ltd.) $10 \mathrm{mg} 3$ times/day.

The 44 patients in the Chinese medicine group were treated with plum blossom needle acupuncture with qiinvigorating superficies-consolidating therapy according to the following protocol: (I) plum blossom needle acupuncture: the operation is performed by the doctors in our hospital. First, the patient's hair loss area was routinely disinfected with $75 \%$ alcohol, and then the scalp was evenly 
coated with a hair growth tincture preparation produced by our hospital (contents: cacumen biotae, Rhizoma drynariae, Salvia miltiorrbiza, white mulberry bark, and Zanthoxylum bungeanum). Wrist force was used to gently pierce the patient's scalp in the hair loss area with the plum blossom needles until the skin began to bleed lightly. The treatment was performed every other day for 10 minutes at a time for 10 sessions total. (II) qi-invigorating superficiesconsolidating therapy: astragalus $30 \mathrm{~g}$, saponin $10 \mathrm{~g}$, forsythia $10 \mathrm{~g}$, poria $10 \mathrm{~g}$, aquilaria $10 \mathrm{~g}$, stone acorus $5 \mathrm{~g}$, acacia peel $30 \mathrm{~g}$, Platycodon grandiflorum $10 \mathrm{~g}$, Chuanxiong rhizome $10 \mathrm{~g}$, Lignum millettiae $30 \mathrm{~g}$, cork $5 \mathrm{~g}$, Tribulus terrestris $15 \mathrm{~g}$, Bauhinia peel $15 \mathrm{~g}$, charred hawthorn 15 g, mulberry 15 g, Polygonum multiflorum $30 \mathrm{~g}$, Radix sileris $15 \mathrm{~g}$, and fried atractylodes $10 \mathrm{~g}$. The patients received 1 dose per day for a total of 14 doses, and the traditional Chinese medicines were decocted and taken orally 3 times per day in the morning, at noon, and in the evening.

Patients in both groups continued to take medication for two months. During the treatment period, both groups of patients were not treated with drugs other than those administered in this study. During the treatment, the patients maintained a light diet and exercised appropriately.

\section{Observation indicators}

The treatment effects were compared between the two groups of patients and classified according to the following characteristics: significantly effective: after 2 months of treatment, the patient's symptoms, including scaling, greasiness, and itching, were alleviated, and more than $60 \%$ of the hair loss area grew new hair. Effective: After 2 months of treatment, the patient's symptoms, including scaling, greasiness, and itching, were alleviated, and more than $30 \%$ of the hair loss area grew new hair. Not effective: The symptoms of scaling, greasiness, and itching were not alleviated, and there was no new hair growth in the hair loss area (6).

The levels of estradiol (E2) and testosterone (T) before and after treatment were compared between the two groups of patients. Because SA is an androgen-related disease, E2 and $\mathrm{T}$ were used as sex hormone evaluation indicators in the study. Five milliliters of fasting venous blood was collected in the morning from each patient before treatment and 2 months after treatment, centrifuged at 2,000 r/min for $15 \mathrm{~min}$, and the supernatant was collected and stored in a refrigerator at $-20^{\circ} \mathrm{C}$ for examination. The $\mathrm{E} 2$ and $\mathrm{T}$ levels in the serum were measured by electrochemiluminescence immunoassay.
The scores of various body signs (hair growth and hair loss status) before and after treatment were compared between the two groups of patients. (I) Hair loss: minimal hair loss ( $<100$ hairs) was scored as 0 points; loss of $\geq 100$ hairs but $\leq 150$ was considered a small amount of hair loss and was scored as 2 points; loss of $\geq 150$ hairs but $\leq 200$ was considered moderate and scored as 4 points; and loss of $\geq 200$ hairs was considered severe and scored as 6 points. (II) Hair growth: if after 2 months of treatment, the entire hair loss areas grew new hair, and hair thickness and color had returned to normal, 0 points; if more than $2 / 3$ of the hair loss areas had new hair growth, but hair color and thickness were not uniform, 2 points; if $1 / 3$ of the hair loss area had new hair growth, and the hair color was pale or the texture was soft, 4 points; if there was no new hair growth or a few hairs, 6 points. The worse the hair growth, the higher the score. The above evaluations were performed after the patients washed their hair in the morning (7).

\section{Adverse reactions in the two groups of patients}

This study mainly evaluated the sexual dysfunction and emotional response of the two groups during the course of treatment. Through consultation, we recorded in the medical record whether the patient had sexual dysfunction, such as decreased sexual desire and male erectile dysfunction. In addition, the patients' emotional changes after medication were recorded, such as anxiety, depression, and hyperactivity.

\section{Statistical analysis}

Data were analyzed using SPSS 25.0 statistical software. Measurement data are represented as $\bar{x} \pm \mathrm{s}$ and were analyzed using the $t$ test. Count data are represented as $\mathrm{n}(\%)$ and were analyzed using the $\chi^{2}$ test. $\mathrm{P}<0.05$ indicates statistical significance.

\section{Results}

\section{Comparison of treatment effects and adverse reactions between the two groups of patients}

The total effective rate of the Chinese medicine group was higher than that of the Western medicine group ( $95.45 \%$ vs. $81.40 \%)$. The incidence of adverse reactions, emotional disorders and sexual dysfunction was lower in the Western medicine group. The differences were statistically significant $(\mathrm{P}<0.05)($ Table 1). 
Table 1 Comparison of treatment effects and adverse reactions between the two groups of patients

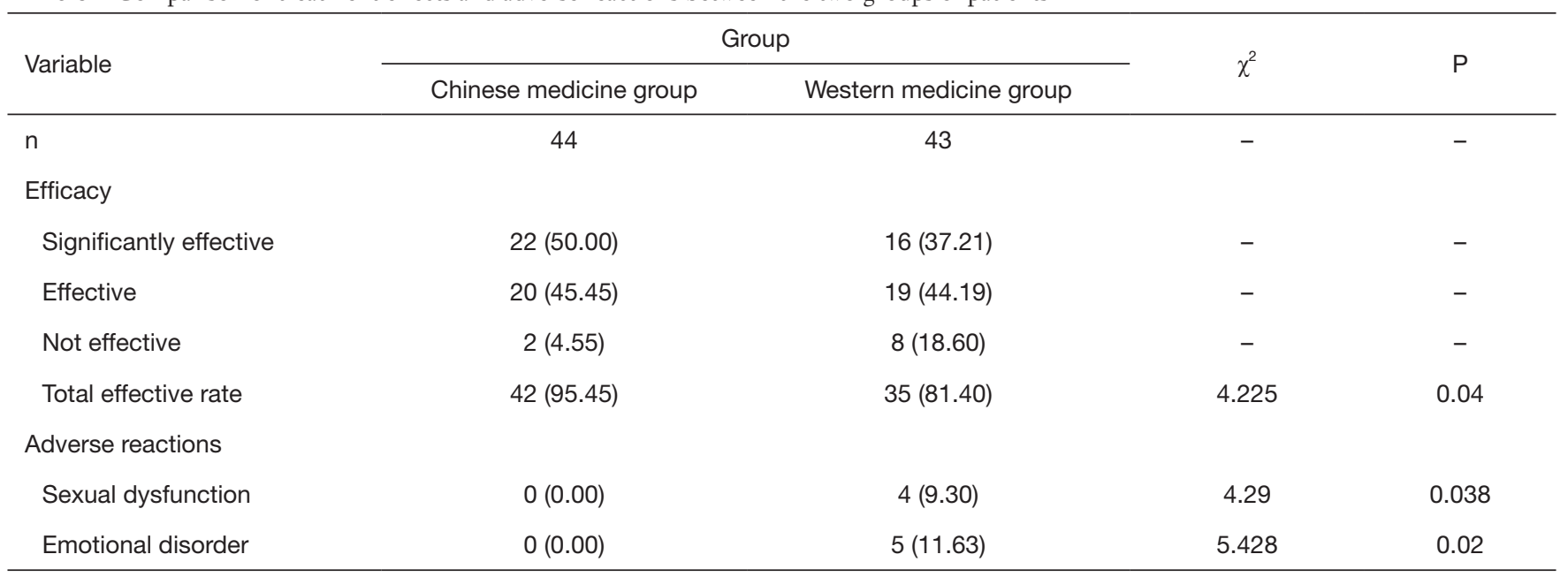

Table 2 Comparison of estradiol (E2) and testosterone (T) levels before and after treatment between the two groups of patients $(\bar{x} \pm \mathrm{s})$

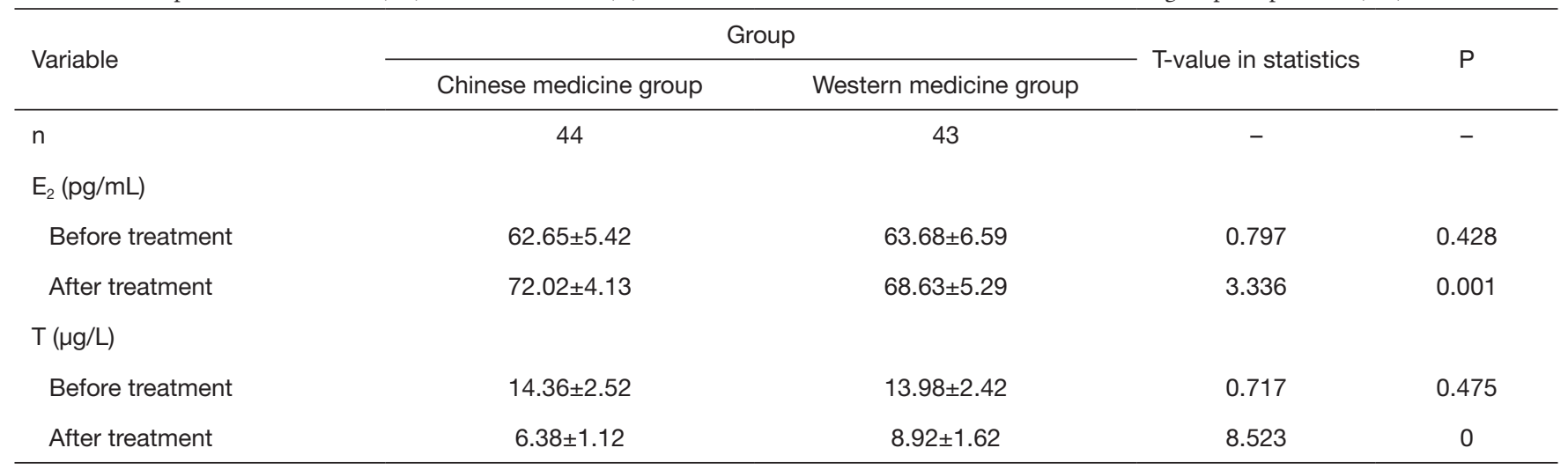

\section{Comparison of estradiol (E2) and testosterone (T) levels} before and after treatment in the two groups of patients

Before treatment, there was no significant difference in the $\mathrm{E} 2$ and $\mathrm{T}$ levels between the two groups $(\mathrm{P}>0.05)$. After treatment, the $\mathrm{T}$ levels of both groups were decreased compared to the levels before treatment, and the E2 levels were higher than those before treatment. The differences between the two groups after treatment were statistically significant $(\mathrm{P}<0.05)$ (Table 2).

\section{Comparison of the changes in various body signs before and after treatment between the two groups of patients}

Before treatment, there were no significant differences in hair growth and hair loss scores between the two groups $(\mathrm{P}>0.05)$. After treatment, the hair growth and hair loss scores of the two groups were lower than before treatment, and those of the Chinese medicine group were lower than those of the Western medicine group; the differences were statistically significant $(\mathrm{P}<0.05)$ (Table 3).

\section{Discussion}

SA is considered a type of "ringworm of the scalp" and "insect bitten alopecia androgenetic alopecia" in traditional Chinese medicine. According to modern medicine, this disease is related to abnormal androgen metabolism in patients and is caused by significantly increased androgen receptors, such us testosterone and dihydrotestosterone, and reductase level; it is also influenced by heredity (8). Traditional Chinese medicine considers Zheng Xu Xie Gan an important factor in the pathogenesis of this disease. 
Table 3 Comparison of the changes in various body signs before and after treatment between the two groups of patients ( $\bar{x} \pm \mathrm{s}$, points)

\begin{tabular}{lccc}
\hline \multirow{2}{*}{ Variable } & \multicolumn{2}{c}{ Group } & T-value in statistics \\
\cline { 2 - 4 } $\mathrm{n}$ & Chinese medicine group & Western medicine group & - \\
Hair growth & 44 & 43 & \\
Before treatment & $5.86 \pm 0.18$ & $5.81 \pm 0.15$ & 3.406 \\
After treatment & $2.21 \pm 1.16$ & $3.56 \pm 1.26$ & 0.163 \\
Hair loss & & & 0 \\
Before treatment & $3.86 \pm 1.36$ & $3.91 \pm 1.22$ & 0.201 \\
After treatment & $1.69 \pm 0.96$ & $2.65 \pm 1.06$ & 0.18 \\
\hline
\end{tabular}

The "Huangdi Neijing" states that "when the evil gathers together, the Qi must be deficient, and when the righteous Qi is stored inside, the evil cannot intervene." Zheng Xu Xie Gan is an important basis for hair loss. Moreover, traditional Chinese medicine believes that this disease is primarily caused by local meridian obstruction, hair root vacuity, and qi and blood disorders. Therefore, reconciling qi and blood and dredging meridians are important methods for the treatment of SA (9).

In the past, patients were often treated with oral drugs and topical ointments in the clinic practice to promote hair follicle regeneration in the area of hair loss, thereby effectively preventing hair loss and promoting the growth of new hair. However, the long-term effect is not very significant; this method can significantly relieve hair loss but has no significant effect on promoting hair growth (10). Therefore, in this study, the method of plum blossom needling was combined with oral administration of traditional Chinese medicine to treat the disease both internally and externally and synergistically address the etiology of the disease. Plum blossom needle acupuncture can effectively promote blood circulation in the hair loss area, reconcile qi and blood, enable qi and blood to flow more smoothly, promote hair growth, stimulate atrophied hair follicles in the hair loss area to restore function as soon as possible, prevent hair follicles from entering the stationary growth phase, and regulate the function of the whole body's neurohumoral system to accelerate the growth of new hair $(11,12)$. These effects were confirmed in this study. In this study, the total effective rate of the Chinese medicine group was significantly higher than that of the Western medicine group (95.45\% vs. $81.40 \%$ ), and the difference was statistically significant $(\mathrm{P}<0.05)$. These results suggest that plum blossom needling combined with qi invigoration superficies-consolidating therapy can achieve significant results in the treatment of SA, improving the symptoms of hair loss and itching and promoting new hair growth. The reason for this effect is that the qiinvigorating superficies-consolidating therapy can nourish kidney qi, reconcile blood and qi, and thus promote the growth of new hair. The Polygonum multiflorum and mulberry in the preparation have the functions of darkening the hair, brightening the eyes and nourishing the liver and kidney. These two components are commonly used to treat white hair and hair loss. Platycodon grandiflorum can induce other medical components to move upward to act on the skin and hair. Bauhinia and Tribulus terrestris have the effects of stopping itching and dispelling evil qi. Atractylodes macrocephala and Poria cocos have the effects of drying dampness and water, invigorating qi and strengthening the spleen. Acorus gramineus has the effects of resolving dampness and enhancing appetite. The combination of various components can enrich the vitality of the kidneys, solidify the surface, and disperse evil qi, which enables the patient's blood to flow unobstructed and encourages more new hair growth. The results are consistent with previous research (13). Modern medicine believes that this disease is related to androgen abnormalities. Therefore, regulating hormone levels is also an important treatment mechanism. In this study, after treatment, the $\mathrm{T}$ levels of both groups were lower than before treatment, and the E2 levels of both groups were higher than before treatment; the differences between the two groups were statistically significant $(\mathrm{P}<0.05)$, suggesting that plum blossom needle acupuncture combined qi-invigorating superficies-consolidating therapy can effectively improve androgen abnormalities in patients 
with SA. The reason for this effect is that plum blossom needle acupuncture has the effects of moistening dryness and stopping itching, nourishing the blood and promoting blood circulation, and regulating the functions of the spleen and stomach; additionally, it can effectively regulate the hypothalamus-pituitary-ovarian axis and hormone secretion. In this study, after treatment, the hair growth and hair loss scores of both groups of patients were lower than before treatment, the scores of the Chinese medicine group were lower than those of the Western medicine group, and the differences were significant $(\mathrm{P}<0.05)$. These results suggest that plum blossom needle acupuncture combined with qi-invigorating superficies-consolidating therapy can effectively promote hair growth and relieve the severity of hair loss in patients with SA. The reason for this effect is that by piercing of the patient's skin, the plum needle acupuncture can adjust the visceral deficiencies and excesses, thus reconciling qi and blood, reconciling yin and yang, and clearing the meridians, and it can effectively promote local blood circulation and reconcile the Yingwei qi and blood of the human body to promote hair growth (14). Modern medicine also believes that there are microcirculatory issues and abnormal blood changes in the skin lesions of the hair loss area. Therefore, promoting blood circulation is also important method for treating this disease. Plum blossom needle acupuncture of the hair loss area can play a role in clearing the meridians, improving qi and blood, promoting blood circulation, promoting the improvement of hair follicles with impaired blood circulation, and promoting hair regeneration.

The course of this disease is often long; patients usually seek medical help many times, and the treatment effect is not ideal. The disease affects patients' appearance and easily leads to depression, anxiety and other emotional disorders and significantly reduced quality of life (15). Therefore, treatments for this disease should not aim for quick or short-term success. Instead, the treatment should take place step by step to regulate emotions and correct bad habits. Western medicine treatments have adverse effects, such as sexual dysfunction and emotional disorders. The treatment period is long, and the disease can easily relapse. The incidence of adverse reactions such as emotional disorders and sexual dysfunction in the Chinese medicine group were lower than those in the Western medicine group, and the difference was statistically significant $(\mathrm{P}<0.05)$, suggesting that the treatment of SA using plum blossom needle acupuncture combined with qi-invigorating superficiesconsolidating therapy has a high degree of safety and few adverse reactions.

In summary, plum blossom needle acupuncture combined with qi-invigorating consolidating superficies therapy for the treatment of SA achieves significant effects, can effectively adjust patients' hormone levels, improves hair loss, and promotes hair growth. This treatment method has the advantages of simple implementation, high tolerance, and low cost and is worth promoting.

\section{Acknowledgments}

Funding: None.

\section{Footnote}

Reporting Checklist: The authors have completed the STRICTA-CONSORT Extension for Acupuncture Checklist. Available at http://dx.doi.org/10.21037/apm20-909

Data Sharing Statement: Available at http://dx.doi. org/10.21037/apm-20-909

Conflicts of Interest: All authors have completed the ICMJE uniform disclosure form (available at http://dx.doi. org/10.21037/apm-20-909). The authors have no conflicts of interest to declare.

Ethical Statement: The authors are accountable for all aspects of the work in ensuring that questions related to the accuracy or integrity of any part of the work are appropriately investigated and resolved. The patients volunteered to participate in this study, and the study was approved by our ethics committee.

Open Access Statement: This is an Open Access article distributed in accordance with the Creative Commons Attribution-NonCommercial-NoDerivs 4.0 International License (CC BY-NC-ND 4.0), which permits the noncommercial replication and distribution of the article with the strict proviso that no changes or edits are made and the original work is properly cited (including links to both the formal publication through the relevant DOI and the license). See: https://creativecommons.org/licenses/by-nc-nd/4.0/.

\section{References}

1. Gao X, Li C, Wang L, et al. Quzhi germinal capsule in the 
treatment of seborrheic alopecia. Journal of Changchun University of Chinese Medicine 2016;32:146-7.

2. Wang Y, Wang W. Clinical Observation of 100 Cases of Seborrheic Alopecia Treated by Dispelling Wind, Tonifying Spleen and Dispelling Dampness Therap. Journal of Sichuan Traditional Chinese Medicine 2015;33:128-9.

3. Jia H, Li L. Clinical study of Erzhiwan combined with finasteride in the treatment of seborrheic alopecia. Modern Journal of Integrated Traditional Chinese and Western Medicine 2019;28:2684-6.

4. Zhang Z. Dermatology of Integrated Traditional Chinese and Western Medicine. Beijing: People's Medical Publishing House, 2000.

5. Yang Y, Liu S, Huang Y, et al. Clinical Efficacy of Dandelion in the Treatment of Alopecia Seborrheica with Spleen Deficiency and Dampness-Heat. Journal of Hebei Traditional Chinese Medicine and Pharmacology 2017;32:11-13.

6. Ling 1, Yang Y, Huang Y, et al. Clinical Efficacy of Compound Cacumen Platycladi Solution in Treating Seborrheic Alopecia. Journal of Guangzhou University of Traditional Chinese Medicine 2019;36:349-352.

7. Zhang X, Zhou D. Clinical effect of Qushi Jianfa decoction combined with plum blossom needle acupuncture in the treatment of alopecia seborrheica with dampnessstagnancy syndrome due to spleen deficiency. China Medical Herald 2019;16:126-9.

8. Mapar MA, Shahriari S, Haghighizadeh MH. Efficacy of platelet-rich plasma in the treatment of androgenetic (male-patterned) alopecia: A pilot randomized controlled

Cite this article as: Li Q, Xie Y, Zha X. The clinical effect of plum blossom needle acupuncture with qi-invigorating superficies-consolidating therapy on seborrheic alopecia. Ann Palliat Med 2020;9(3):1030-1036. doi: 10.21037/apm-20-909 trial. J Cosmet Laser Ther 2016;18:452-5.

9. Kong $\mathrm{Y}$, Wang Z, Wang C, et al. Observation on the Efficacy of Acupuncture plus Medication in Treating Male Seborrheic Alopecia Due to Damp-heat Steaming. Shanghai Journal of Acupuncture and Moxibustion 2019;38:436-40.

10. Ayatollahi A, Hosseini H, Shahdi M, et al. Plateletrich Plasma by Single Spin Process in Male Pattern Androgenetic Alopecia: Is it an Effective Treatment? Indian Dermatol Online J 2017;8:460-4.

11. Wu S, Li Y. Professor Li Yongmei's cream method for the treatment of seborrheic alopecia. Chinese Journal of Dermatovenerology of Integrated Traditional and Western Medicine 2018;17:546-9.

12. Xiang J, Bian X. Therapeutic Observation of SHI's Needle Warming Therapy plus Chinese Medication for Alopecia Seborrheica. Shanghai Journal of Acupuncture and Moxibustion 2018;37:544-7.

13. Chen Y, Kuang L, Huang E. Clinical Observation of Quzhi Shengfa Liquid Combined with Minoxidil in Treatment of Seborrheic Alopecia. Journal of Traditional Chinese Medicine University of Hunan 2017;37:1285-7.

14. Zhong C, Zhang Z, Liu C, et al. TCM master XUAN Guo-wei's experience in treating alopecia seborrheica. China Journal of Traditional Chinese Medicine and Pharmacy 2018;33:133-5.

15. Wang Y, Zhang Q. Efficacy of Quzhi Shengfa decoction combined with finasteride on splenogastric moist heat-type seborrheic alopecia and the effect on trace element levels in hair. Modern Journal of Integrated Traditional Chinese and Western Medicine 2018;27:1338-40. 